\title{
Posterior cortical lesions and specific crossmodal transfer in the rat
}

\author{
EDWARD H. YETERIAN, ROBERT S. WATERS, and WILLIAM A. WILSON, JR. \\ University of Connecticut, Storrs, Connecticut 06268
}

\begin{abstract}
The present study investigated the neural substrate of specific crossmodal transfer (CMT) in rats, using a direct vs. reversal transfer paradigm. Animals with posterior cortical ablations or sham operations were first trained to criterion on a visual intensity discrimination. Following completion of visual training, they received an auditory intensity discrimination (transfer). A factorial design was used, involving operated groups, intensity of visual S+, and intensity of auditory S+. Among sham operates, both direct transfer subgroups showed performance superior to their corresponding reversal transfer subgroups early in the auditory modality, indicating the presence of specific CMT. Among cortical operates, no direct-reversal differences were found. The results demonstrate the importance of the functional integrity of posterior neocortex of the rat to specific CMT involving the modalities of vision and audition.
\end{abstract}

There is now a large body of evidence concerning the different neural systems involved in modality-specific learning (e.g., learning based on visual cues, or on auditory cues, etc.). However, investigations of brain mechanisms involved in interactions between the various sensory modalities have been few in number. In particular, with the exception of a single study using monkeys (Saghal, Petrides, \& Iversen, 1975), there is almost no direct evidence concerning the brain mechanisms necessary for specific crossmodal transfer (CMT), a phenomenon which has been considered to be fundamental to the development of language (e.g., Geschwind, 1964). Studies of specific CMT determine whether stimulus information from one modality is utilized in responding to stimuli in an alternative modality. Specific CMT paradigms do not assume necessarily that stimuli per se are identical across modalities, although nominally identical stimuli (e.g., identical rates of intermittence for rhythmic visual and auditory stimuli) often are used. Rather, the relationships between discriminative stimuli in each modality must be analogous for performance in the second (transfer) modality to reflect stimulusreinforcement contingencies learned in the first. For example, Over and Mackintosh (1969) have shown that rats trained to discriminate between high and low intensities of light, or of sound, have a significant tendency to respond in a similar manner (to high vs. low) when presented with stimuli differing along the intensity dimension in the other modality. In the present study,

This study is based on a paper presented at the meeting of the American Psychological Association, New Orleans, September 1974. This research was supported by USPHS Grant MH10972 to Dr. W. A. Wilson, Jr. The present address of the senior author is Harvard Neurological Unit, Beth Israel Hosptial, 330 Brookline Ave., Boston, Massachusetts 02215. Requests for reprints should be sent to E. H. Yeterian, Harvard Neurological Unit, Beth Israel Hospital, 330 Brookline Ave., Boston, Massachuset ts 02215. this paradigm was used to begin an exploration of the neural substrates of specific CMT in rats.

\section{METHOD}

Subjects

Sixteen male black-hooded rats (Long-Evans derived), obtained from the breeding colony of the University of Connecticut Department of Psychology, were used. The rats were housed in individual cages in a continuously lit room. Two squads of eight each were run, and the animals were from 120 to 150 days old at the beginning of training.

\section{Apparatus}

A $25 \times 25 \times 30 \mathrm{~cm}$ operant chamber, placed in a soundreducing enclosure and controlled by conventional relay equipment, was used. A 9-cm lever, mounted directly above the foodcup, served as the manipulandum. The only illumination in the chamber was that provided by incandescent bulbs mounted above a translucent white Plexiglas roof. Variations in their intensity gave the discriminative visual stimuli of 14 and $0.05 \mathrm{fc}$, measured $5 \mathrm{~cm}$ above the floor. The ambient temperature of the enclosure increased approximately $2.4^{\circ} \mathrm{C}$ during a session, but there were no increases or decreases systematically related to the high- or low-intensity light conditions. During preliminary training and during auditory transfer, the light was at $0.6 \mathrm{fc}$. To the accuracy of measurement, these values were equal to those of Over and Mackintosh. The auditory stimuli were two intensities, 95 and $66 \mathrm{~dB}$ SPL, of white noise played through a loudspeaker in the enclosure, and the noise was at $82 \mathrm{~dB}$ during preliminary and visual training. The values used by Over and Mackintosh were, respectively, 95,62 , and $79 \mathrm{~dB}$.

\section{Procedure}

Preliminary leverpress training. The rats were reduced to $80 \%$ of ad-lib body weight for preliminary training. With the light and the sound at middle intensities, they were shaped to press the lever for 45 -mg food pellets (Noyes) on a continuous reinforcement schedule, taking from 1 to 3 days to reach a criterion of 50 presses in a single session. Then, over a period of 2 days, intermittent schedules were introduced. On the last training day before surgery, each animal had $10 \mathrm{~min}$ of VI $5 \mathrm{sec}$ and $35 \mathrm{~min}$ of VI $12 \mathrm{sec}$, with a criterion of 300 or more responses within the 45-min session.

Surgery and histology. After several days of ad-lib feeding, 
all animals received a surgical procedure, ablation of posterior neocortex or a sham operation. The animals received $0.1 \mathrm{cc}$ atropine sulfate $(1 \mathrm{mg} / \mathrm{cc})$ preoperatively and $0.1 \mathrm{cc}$ Duracillin immediately postoperatively. The lesions were produced by suction, with the rat anesthetized with pentobarbital sodium $(60 \mathrm{mg} / \mathrm{kg} \mathrm{IP})$ and held in a stereotaxic frame equipped with hollow ear bars. The intended lesion included all cortex posterior to bregma, laterally to the rhinal fissure. The control subjects were anesthetized and placed in the frame, and the scalp was cut and retracted as for the lesion operation, but no intrusion into the skull was made.

Upon completion of the experiment, the cortical operates were sacrificed by means of saline-Formalin perfusion. Extents of cortical removal were recorded on Lashley brain diagrams; the smallest and largest of the removals are shown in Figure 1.

Postoperative training. After a recovery period of 7 to 10 days, the animals were returned to $80 \%$ body weight. Additional pretraining consisted of 64-min sessions on a VI 12-sec schedule. Sessions were given daily until an animal made at least 300 responses in a session; this took no longer than 2 days for any subject. Immediately following the last day of pretraining, all rats received training on a visual intensity discrimination. The experimental sessions were 64 min long, and each consisted of alternating periods of positive ( $\mathrm{S}+$ ) and negative (S-) stimuli. Within each of eight 8-min blocks, there were two periods of $60 \mathrm{sec}$ and one each of 30 and $90 \mathrm{sec}$ for each stimulus. The sequences were balanced within each 8-min block, and the blocks were balanced over days. During periods of $S+$, leverpresses were rewarded on a VI 24-sec schedule; during S-, no reinforcement could be gained. Training on the visual discrimination problem was continued until a given subject's response rate during $S+$ was at least six times that during $\mathrm{S}$ - for a single session, or until it had completed 25 sessions. Upon completing visual training, each animal received daily sessions of an auditory discrimination until they attained a criterion $S+/ S$ - ratio of 6.0 or greater, or until they had been run for 25 sessions. Training was given 6 days per
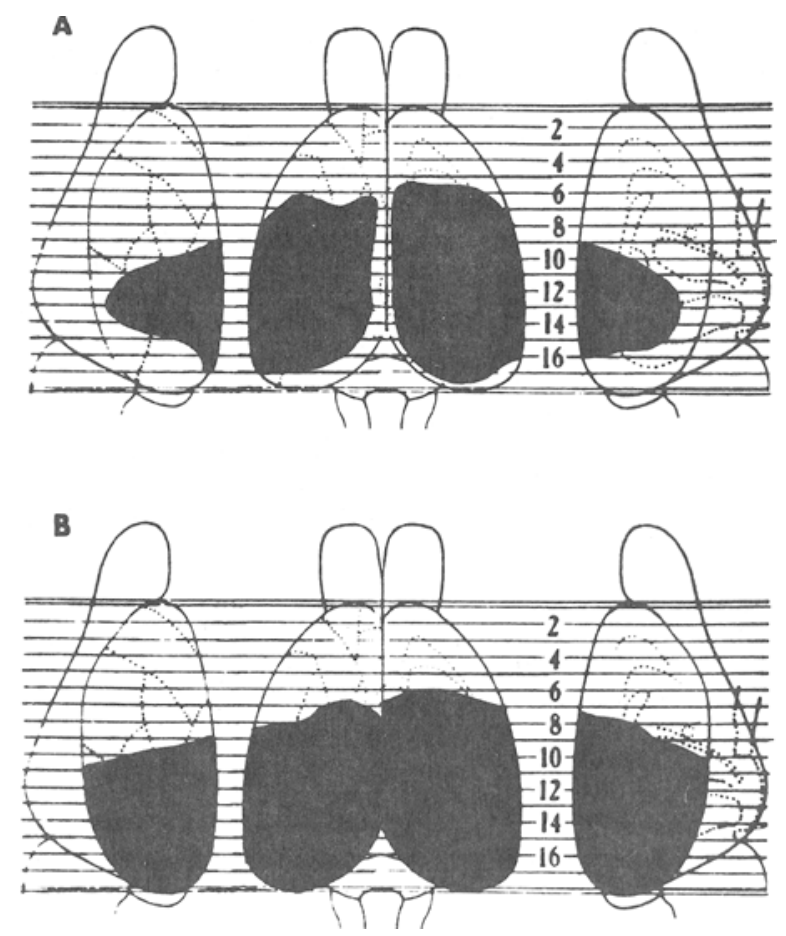

Figure 1. Lashley diagrams showing the smallest (A) and largest (B) amount of bilateral cortical removal. week, with the exception that the first session of auditory transfer always came on the day immediately following the last day of visual training.

A factorial design was used, involving operated groups, intensity of visual S+, and intensity of auditory S+. Within each operated subgroup, in visual discrimination training, half of the animals received the high-intensity stimulus as $\mathrm{S}+$, while the other half received low-intensity as $\mathrm{S}+$. In auditory transfer, the high and low visual $\mathrm{S}+$ animals again were divided in half, into those receiving high or low auditory S+. Thus, within each operated group, there were two "direct transfer" subgroups (high visual $\mathrm{S}+$ followed by high auditory $\mathrm{S}+$, and low followed by low), and two "reversal transfer" subgroups (high visual S+ followed by low auditory S+, and low followed by high). The presence of specific CMT among animals in either of the operated groups was assessed by comparing the performance early in the auditory modality of pairs of direct and reversal subgroups which had the same S+ intensity in the transfer modality. The rationale of the direct-reversal paradigm for specific CMT has been discussed in detail by Ward, Yehle, and Doerflein (1970).

\section{RESULTS}

The mean proportions of responses to $\mathrm{S}+$ are shown in Figure 2 for the subgroups of sham operates and of animals with cortical lesions, for each of the eight 8-min blocks on the critical first day of the transfer modality. The sham operates show three main effects: (1) Specific CMT is seen as the superiority of the animals that received the same $\mathrm{S}+$ (either high or low) in the two modalities (e.g., O . . . O vs. X ... X. (2) An incidental stimulusintensity effect is seen as the superiority of animals that received the high sound level as $\mathrm{S}+$ (e.g., $\mathrm{O} \ldots \mathrm{O}$ vs. $\mathrm{O}-0$ ). This effect has been observed also by Over and Mackintosh early in auditory training of naive animals; therefore it is not related systematically to the sequence of visual training followed by auditory transfer. (3) Finally, there is rapid learning in the new modality, seen as general improvement over the session. None of these findings is present among the animals with lesions. Furthermore, neither the transfer effect nor the stimulus-intensity difference appeared in the lesion group on any of the subsequent days of the auditory discrimination.

Following Over and Mackintosh, analysis of variance was carried out on the first four 8-min intervals. The difference between the surgical groups is supported by an interaction between Transfer and Lesion $(F=11.46$, $\mathrm{df}=1 / 8, \mathrm{p}<.01)$. Performance depends upon the intensity of the auditory $\mathrm{S}+(\mathrm{F}=29.34, \mathrm{df}=1 / 8, \mathrm{p}<.01)$; for sham operates, the high $\mathrm{S}+$ produces the greater proportion of responses to $S+$ (interaction $F=51.51$, $\mathrm{df}=1 / 8, \mathrm{p}<.01$ ). Also significant are the effect of Blocks $(\mathrm{F}=8.03, \mathrm{df}=3 / 24, \mathrm{p}<.01)$ and the interaction between Blocks and S+ Intensity $(F=3.52, \mathrm{df}=3 / 24$, $\mathrm{p}<.05)$. Separate analyses of the operated groups confirmed these conclusions. The shams show significant effects of Transfer $(F=8.19, \mathrm{df}=1 / 4, \mathrm{p}<.05)$, Auditory S+ $(\mathrm{F}=46.49, \mathrm{df}=1 / 4, \mathrm{p}<.01)$, and Blocks $(F=6.41, d f=3 / 12, p<.01)$, while no main effects 

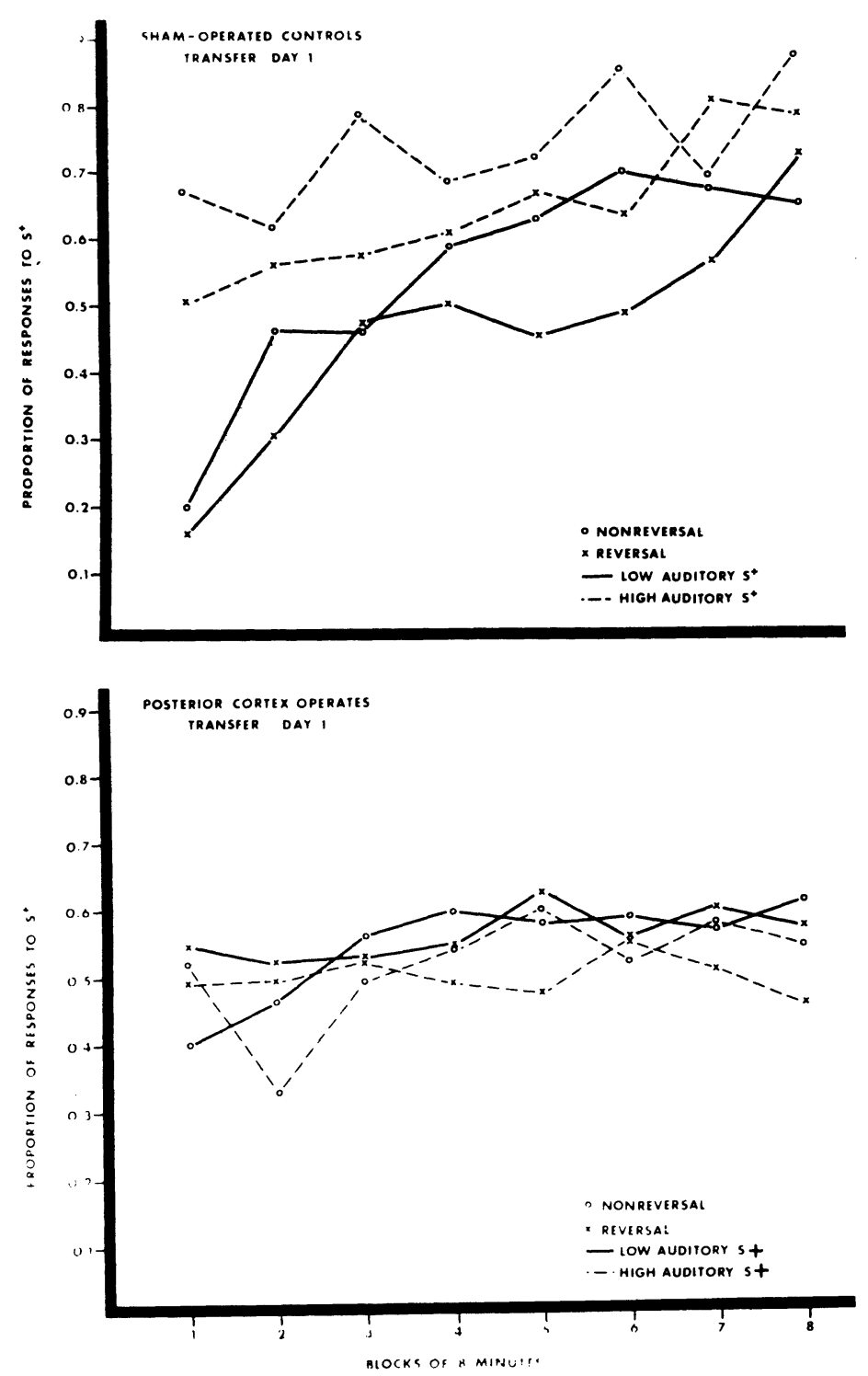

Figure 2. Mean proportions of S+ responses for the various subgroups of rats with sham operations (above) and cortical lesions (below), for 8-min intervals on the first day of transfer to the auditory task. or interactions meet the .05 level for the animals with lesions. The difference between the Direct and Reversal Transfer sham subgroups in proportions of responses to S+ dissipates by the end of the first day of transfer. As this fact would suggest, no difference was found between these two subgroups (nor the comparable lesion subgroups) when numbers of days to reach criterion were compared.

The operates as a group were slower to reach criterion on the discrimination tasks than the sham animals. Median numbers of days to criterion were, respectively, 22 and 9 (Mann Whitney $U=10.5, p<.05$ ) on the visual problem, and 22 and $8(U=6.5, p<.01)$ on the auditory discrimination. Three of the cortical operates and one of the shams failed to meet the criterion of $\mathrm{S}+/ \mathrm{S}-\geqslant 6.0$ on the visual discrimination, but all of these animals formed a discrimination (with an $\mathrm{S}+/ \mathrm{S}$ - ratio of at least 2.5) and appeared to have reached an asymptote.
Furthermore, the behavior of these animals on the first day of transfer did not differ from that of the corresponding animals that met the $\geqslant 6.0$ standard. The mean proportions of responses to $\mathrm{S}+$ during the first half of Transfer Day 1 for the animals with cortical lesions were .46 for $\mathrm{Hi} \mathrm{V} \rightarrow \mathrm{Hi} \mathrm{A}, .49$ for Lo V $\rightarrow \mathrm{Hi} \mathrm{A}$, .50 for $\mathrm{Lo} \mathrm{V} \rightarrow \mathrm{Lo} \mathrm{A}$, and .52 for $\mathrm{Hi} \mathrm{V} \rightarrow$ Lo A. If the rats that failed to reach the $\geqslant 6.0$ criterion are excluded, the comparable figures are $.46, .50, .53$, and .52 respectively.

\section{DISCUSSION}

The sham operates demonstrate that the crossmodal transfer reported by Over and Mackintosh is a reproducible phenomenon; the results with the operated rats show that this phenomenon depends upon the integrity of the posterior neocortex. The difference in transfer 
between the surgical groups is not a consequence of the difference in overall rate of learning of the individual discrimination tasks. When animals with identical experimental histories were examined, there was no evidence of a correlation between speed of learning of the visual or the auditory task and performance on the first day of transfer. The lack of improvement of the operated rats on Transfer Day 1, with eventual improvement over days, is similar to the performance generally exhibited by naive normal animals on either an auditory or a visual task using the present paradigm.

Over and Mackintosh discuss some possible reasons why they were able to demonstrate specific CMT of intensity in rats even though Wegener (1965) had not seen it in monkeys. While those factors may be important indeed, they become of less immediate concern when we note that Wegener has recently reexamined his data and found evidence for transfer early in the first day on the new modality (Frampton, Milner, \& Ettlinger, 1973). Taken with the earlier (Wilson \& Shaffer, 1963) and more recent (Cowey \& Weiskrantz, 1975; Frampton et al., 1973) positive findings on monkeys, the replicable results with auditory-visual rate discrimination transfer in bushbabies (Ward et al., 1970; Ward, Vise, \& Frank, Note 1; Ward \& Frank, Note 2), the strong effects in visual-haptic form transfer in apes (e.g., Davenport, Rogers, \& Russell, 1973), etc., these results suggest that it is time to acknowledge that specific CMT is widespread across species and turn our attention to more interesting questions of mechanism and function.

Lesions of modality-specific association cortex in monkeys have been shown to produce deficits in crossmodal matching to sample (Saghal et al., 1975), and in crossmodal transfer of a very general nature, namely of learning set formation (Wilson \& Wilson, 1962). However, lesions of cortex between primary auditory and visual regions in bushbabies did not affect auditoryvisual rate discrimination transfer (e.g., Ward et al., Note 1). In the present study, lesions that involved both visual and auditory cortex eliminated specific CMT from vision to audition, although the lesions did not preclude acquisition of discriminations in the separate modalities. There is ample evidence that visual and auditory activity converges upon cells of the visual cortex in related species, e.g., rabbits (Polyansky, Sokolov, \& Polkoshnikov, 1975). It may be that the destruction of such pathways eliminates a "pre-wired" interconnection between subsystems within the two modalities that carry messages signaling equivalent levels of stimulus intensity. Alternatively, it is possible that these areas of cortex contain an essential portion of a system that is concerned with "forming a supramodal principle involving the differentiation of larger or smaller stimuli" (Over \& Mackintosh, 1969, p. 919). In either case, our results clearly provide no support for a suggestion derived from the work of Gillespie and Cooper (1973): that decorticate rats would do better than controls on CMT because they are deprived of systems important for the analysis of the distinctive nature of the signals of the two modalities.

\section{REFERENCE NOTES}

1. Ward, J. P., Vise. M., \& Frank, J. Crossmodal transfer in the bush bahy Galago senegalensis with lesion of association cortex. Paper presented at the meeting of the Psychonomic Society, St. Louis, November 1972.

2. Ward, J. P., \& Frank, J. Intermodal transfer in the bush baby Galago senegalensis with lesions of posterior neocortex. Paper presented at the meeting of the Suciety for Neuroscience. St. Louis, October 1974.

\section{REFERENCES}

Cowey, A.. \& Weiskrantz, L. Demonstration of cross-modal matching in the rhesus monkey, Macaca mulatta. Neuropsychologia, 1975, 13, 117-120.

Davenport, R. K., Rogers, C. M., \& Russell, I. S. Crossmodal perception in apes. Neuropsychologia, 1973, 11, 21-28.

Frampton, G. B., Milner, A. D., \& Etrlinger, G. Crossmodal transfer between vision and touch of go, no go discrimination learning in the monkey. Neuropsychologia. 1973, 11. 231-233.

Gescrwind, N. The development of the brain and the evolution of language. In C. I. J. M. Stuart (Ed.), Monograph Series on Languages and Linguistics, No. 17, 1964, 155-169.

Gillespie. L. A., \& Cooper, R. M. Visual cortical lesions in the rat and a conditioned emotional response. Journal of Comparative and Physiological Psychology. 1973. 83. 76-91.

OVER, R., \& MACKINTOSH, N. Cross-modal transfer of intensity discrimination by rats. Nature. 1969, 224, 918-919.

Polyansky, V. B.. Sokolov, B. N., \& Polkoshnikov, E. V. Light-sound interactions in the neurons of the rabbit's visual cortex. Acta Neumbiologicae Experimentalis. 1975. 35. $51-76$.

Sahgal. A., Petrides, M., \& Iversen, S. D. Cross-modal matching in the monkey after discrete temporal lobe lesions. Nature. 1975, 257, 672-673.

WARD, J. P., Yehle, A. L., \& Doerflein, R. S. Cross-modal transfer of a specific discrimination in the bush baby (Galago senegalensis). Journal of Comparative and Physiological Psychology, 1970, 73, 74-77.

Wegener. J. G. Cross-modal transfer in monkeys. Joumal of Comparative and Physiological Psychology, 1965, 59, 450-452.

Wilson, W. A.. JR.. \& SHAFfer, O. C. Intermodality transfer of specific discriminations in the monkey. Nature, 1963, 197, 107.

WILSON, M., \& WILSON, W. A.. JR. Intersensory facilitation of learning sets in normal and brain operated monkeys. Journal of Comparative and Physiological Psychology. 1962. 55. 931-9.34.

(Received for publication January 12, 1976; accepted March 2, 1976.) 